\title{
High-resolution DNA-binding specificity analysis of yeast transcription factors
}

Cong Zhu, ${ }^{1,9}$ Kelsey J.R.P. Byers, ${ }^{1,9}$ Rachel Patton McCord, ${ }^{1,2,9}$ Zhenwei Shi, $^{3}$ Michael F. Berger, ${ }^{1,2}$ Daniel E. Newburger, ${ }^{1}$ Katrina Saulrieta, ${ }^{1,4}$ Zachary Smith, ${ }^{1,4}$ Mita V. Shah, ${ }^{1,5}$ Mathangi Radhakrishnan, ${ }^{1,6}$ Anthony A. Philippakis, ${ }^{1,2,7}$ Yanhui $\mathrm{Hu}^{3}$ Federico De Masi, ${ }^{1}$ Marcin Pacek, ${ }^{3}$ Andreas Rolfs, ${ }^{3}$ Tal Murthy, ${ }^{3}$ Joshua LaBaer, ${ }^{3}$ and Martha L. Bulyk ${ }^{1,2,7,8,10}$

${ }^{1}$ Division of Genetics, Department of Medicine, Brigham and Women's Hospital and Harvard Medical School, Boston, Massachusetts 02115, USA; ${ }^{2}$ Committee on Higher Degrees in Biophysics, Harvard University, Cambridge, Massachusetts 02138, USA; ${ }^{3}$ Harvard Institute of Proteomics, Harvard Medical School, Cambridge, Massachusetts 02141, USA; ${ }^{4}$ Department of Biology, Massachusetts Institute of Technology, Cambridge, Massachusetts 02139, USA; ${ }^{5}$ Department of Biology, Wellesley College, Wellesley, Massachusetts 02481, USA; ${ }^{6}$ Department of Biological Engineering, Massachusetts Institute of Technology, Cambridge, Massachusetts 02139, USA; ${ }^{7}$ Harvard/MIT Division of Health Sciences and Technology (HST), Harvard Medical School, Boston, Massachusetts 02115, USA;

${ }^{8}$ Department of Pathology, Brigham and Women's Hospital and Harvard Medical School, Boston, Massachusetts 02115, USA

Transcription factors (TFs) regulate the expression of genes through sequence-specific interactions with DNA-binding sites. However, despite recent progress in identifying in vivo TF binding sites by microarray readout of chromatin immunoprecipitation (ChIP-chip), nearly half of all known yeast TFs are of unknown DNA-binding specificities, and many additional predicted TFs remain uncharacterized. To address these gaps in our knowledge of yeast TFs and their cis regulatory sequences, we have determined high-resolution binding profiles for 89 known and predicted yeast TFs, over more than 2.3 million gapped and ungapped 8-bp sequences ("k-mers"). We report 50 new or significantly different direct DNA-binding site motifs for yeast DNA-binding proteins and motifs for eight proteins for which only a consensus sequence was previously known; in total, this corresponds to over a $50 \%$ increase in the number of yeast DNA-binding proteins with experimentally determined DNA-binding specificities. Among other novel regulators, we discovered proteins that bind the PAC (Polymerase $\underline{A}$ and $\underline{\text { C }}$ ) motif (GATGAG) and regulate ribosomal RNA (rRNA) transcription and processing, core cellular processes that are constituent to ribosome biogenesis. In contrast to earlier data types, these comprehensive $k$-mer binding data permit us to consider the regulatory potential of genomic sequence at the individual word level. These $k$-mer data allowed us to reannotate in vivo TF binding targets as direct or indirect and to examine TFs' potential effects on gene expression in $\sim 1700$ environmental and cellular conditions. These approaches could be adapted to identify TFs and cis regulatory elements in higher eukaryotes.

[Supplemental material is available online at www.genome.org and at http:// thebrain.bwh.harvard.edu/. Gene expression microarray data have been submitted to the Gene Expression Omnibus (GEO) (http:/ / www.ncbi.nlm.nih.gov/geo/) database under accession no. GSE13684. Protein-binding microarray data are available at http:// thebrain.bwh.harvard. edu/ and in the UniPROBE database, http:/ / thebrain.bwh.harvard.edu/ uniprobe/.]

Transcription factors (TFs) are major regulators that control critical cellular processes and responses to environmental conditions. TFs control the expression of their target genes by binding to cis regulatory elements in a sequence-specific manner. Thus, TFs and their DNA-binding sites are of central importance for gene regulation, and intensive efforts have been invested in identifying TF binding sites.

S. cerevisiae is an important model organism in understanding fundamental biological pathways and transcriptional regulatory networks (Ideker et al. 2001; Harbison et al. 2004; MacIsaac et al. 2006; Workman et al. 2006), developing genome sequence anal-

\footnotetext{
${ }^{9}$ These authors contributed equally to this work. ${ }^{10}$ Corresponding author.

E-mail mlbulyk@receptor.med.harvard.edu; fax (617) 525-4705. Article published online before print. Article and publication date are at http://www.genome.org/cgi/doi/10.1101/gr.090233.108. Freely available online through the Genome Research Open Access option.
}

ysis algorithms (Cliften et al. 2003; Kellis et al. 2003) that are subsequently applied to the genomes of higher organisms, and considering regulatory sequence evolution (Gasch et al. 2004; Tanay et al. 2005). However, even in S. cerevisiae, in which transcriptional regulation has been studied extensively both computationally (Hughes et al. 2000a; Cliften et al. 2003; Kellis et al. 2003; Beer and Tavazoie 2004) and experimentally using traditional and high-throughput genomic approaches (for review, see Bulyk 2006), the identities of the TFs that regulate major functional categories of genes or coexpressed genes remain unknown. For example, the PAC motif was identified nearly two decades ago (Dequard-Chablat et al. 1991) and computational studies have associated it with regulation of ribosomal RNA (rRNA) processing genes (Hughes et al. 2000a; Pilpel et al. 2001; Beer and Tavazoie 2004), but the trans factor(s) that bind this motif have remained unknown. A number of studies have utilized chromatin immunoprecipitation (ChIP) followed by microarray (ChIP-chip) (Ren 
et al. 2000; Iyer et al. 2001; Lieb et al. 2001) to experimentally identify genomic regions occupied by TFs in vivo in the examined condition through either direct or indirect association with DNA; however, computational analysis of the binding data from those studies failed to yield sequence-specific binding motifs for half of the $\sim 200$ known yeast TFs (Harbison et al. 2004; MacIsaac et al. 2006).

Most of the available ChIP-chip data on yeast TFs was obtained using array technology that provides TF binding data for entire intergenic regions. More recently, ChIP coupled with higher-density tiling arrays (Pokholok et al. 2005; Borneman et al. 2007) or with new generation sequencing technologies (ChIP-seq [Johnson et al. 2007], ChIP-paired-end diTag [ChIP-PET] [Wei et al. 2006]) has permitted higher resolution identification of in vivo TF binding sites. However, relatively few TFs have been examined by these higher resolution approaches, and the resulting data can still leave a challenge in distinguishing direct binding sites from those bound indirectly. Consequently, the functions and conditionspecific regulatory roles of many known yeast TFs, even those with previously characterized binding specificities, are still not well understood, and many predicted TFs remain uncharacterized. Moreover, even well-studied TFs' DNA-binding preferences are often not sufficiently characterized to be able to accurately assess the consequences of nucleotide substitutions in their known binding sites, and a model of the binding preferences is typically generated based upon only a few dozen known binding sites.

Here we report high-resolution DNA-binding profiles and motifs for 89 TFs, utilizing protein-binding microarray (PBM) technology (Bulyk et al. 2001; Mukherjee et al. 2004) that covers all possible contiguous 8-mers and a large variety of gapped 8-mers (Berger et al. 2006). Briefly, custom-designed oligonucleotide arrays (Philippakis et al. 2008) are converted to double-stranded DNA arrays, incubated with GST-TF fusion protein in an in vitrobinding reaction, stained with fluorophore-conjugated anti-GST antibody, and scanned in a microarray scanner, followed by quantification of array signal intensities and sequence analysis (Berger et al. 2006). The resulting PBM data not only provide comprehensive DNA-binding preferences over all possible DNAbinding site variants, but also identify previously undiscovered DNA-binding proteins and their DNA-binding specificities, including two newly discovered TFs that bind to PAC sites and regulate rRNA processing genes. We predict the potential target genes, regulatory roles, and condition specificities of these TFs using their 8-mer binding profiles. While current PBM technology assesses the direct binding of protein to nucleosome-free DNA on the surface of arrays, PBM data on TFs' direct DNA-binding preferences in vitro are complementary to in vivo ChIP-based studies that provide information on both direct and indirect TF occupancy in particular conditions. We show that PBM data can be used to further interpret ChIP-chip data in order to distinguish likely direct versus indirect binding targets. Our extensive PBM $k$-mer data provide a valuable resource for future studies of transcriptional regulatory networks.

\section{Results}

DNA-binding specificity survey of known and predicted yeast transcription factors

To address major gaps in our knowledge of yeast TFs and their cis regulatory sequences, we have determined the comprehensive DNA-binding sequence specificities of 89 known and predicted yeast TFs using custom-designed universal protein-binding microarrays (PBMs) (Bulyk et al. 1999; Mukherjee et al. 2004; Berger et al. 2006). This data set constitutes the high-confidence data that we obtained from a DNA-binding specificity survey of 246 candidate TFs that we examined in this study (Supplemental Fig. S1, S2; Supplemental Table S1). Our survey included 157 proteins for which we did not obtain $k$-mer binding profiles that met our conservative acceptance criteria (Supplemental Methods). Our criteria for including candidate regulatory proteins in this survey were permissive and likely included proteins that do not bind DNA sequence specifically, if at all. Indeed, 14 of the proteins that did not yield motifs belong to structural classes for which there is no prior evidence for sequence-specific DNA-binding activity (bromodomain, PHD, Sir2, and Zf_CCCH), while another 58 had no identifiable DNA-binding domain and no prior evidence for DNA sequence-specific binding activity. Other factors, including 56 TFs that yielded motifs in previous analysis of ChIP-chip data (MacIsaac et al. 2006), may not have yielded motifs in our PBM survey as our criteria for acceptance of PBM-derived motifs may have been overly conservative. In addition, some TFs may not have been folded properly or may require protein partners, small molecule cofactors, post-translational modifications, particular buffer conditions, or a native chromatin context for sequencespecific DNA binding.

For each TF, from the PBM signal intensity data we calculated the relative sequence preferences, using an enrichment score $(E$ score) that ranges from -0.5 to 0.5 for each of more than 2.3 million gapped and ungapped 8-mers spanning the full affinity range from highest affinity to nonspecific sequences (Berger et al. 2006). These high-resolution $k$-mer binding profiles provide vastly more comprehensive binding specificity data than had been identified previously. In order to examine the landscape of sequence specificity across our entire data set, we performed twodimensional clustering of the TFs' $k$-mer binding profiles (Fig. 1A; Supplemental Fig. S3). In general, the binding profiles of TFs of the same DNA-binding domain structural class were more similar to each other than to the profiles of TFs from different structural classes.

Despite the fact that PBM $k$-mer data provide greater depth on the relative sequence preferences of TFs than do position weight matrices (PWMs) (Berger et al. 2008), in order to represent these DNA-binding specificities compactly, we constructed PWM-based motif representations using our Seed-and-Wobble algorithm (Berger et al. 2006) (Fig. 1B; Supplemental Fig. S4). For most previously characterized TFs, and in particular for the most wellknown TFs, our PBM-derived motifs matched their previously known motifs (Supplemental Table S2). Consistent with prior knowledge of how proteins with a $\mathrm{Zn}_{2} \mathrm{Cys}_{6}$ ("Gal4-type") DNAbinding domain can dimerize and interact with DNA (Reece and Ptashne 1993; Liang et al. 1996; Mamane et al. 1998), most TFs with a $\mathrm{Zn}_{2} \mathrm{Cys}_{6}$ DNA-binding domain have very similar half-site preferences, and appear to derive much of their specificities from the lengths of the degenerate spacers separating their half-sites. However, we found that not all $\mathrm{Zn}_{2} \mathrm{Cys}_{6}$ proteins bind CGG triplets; Rsc3 and Rsc30 appear to bind CGCGCGC and CGCGCGCGCG motifs, respectively.

We report experimentally determined DNA-binding site motifs for 30 known or predicted TFs lacking any prior motif data, and motifs for 11 additional TFs that have only consensus sequences reported in the literature. Among the 30 TFs we characterized that had no previously known DNA-binding specificities were 21 putative TFs, including many of unknown function. 


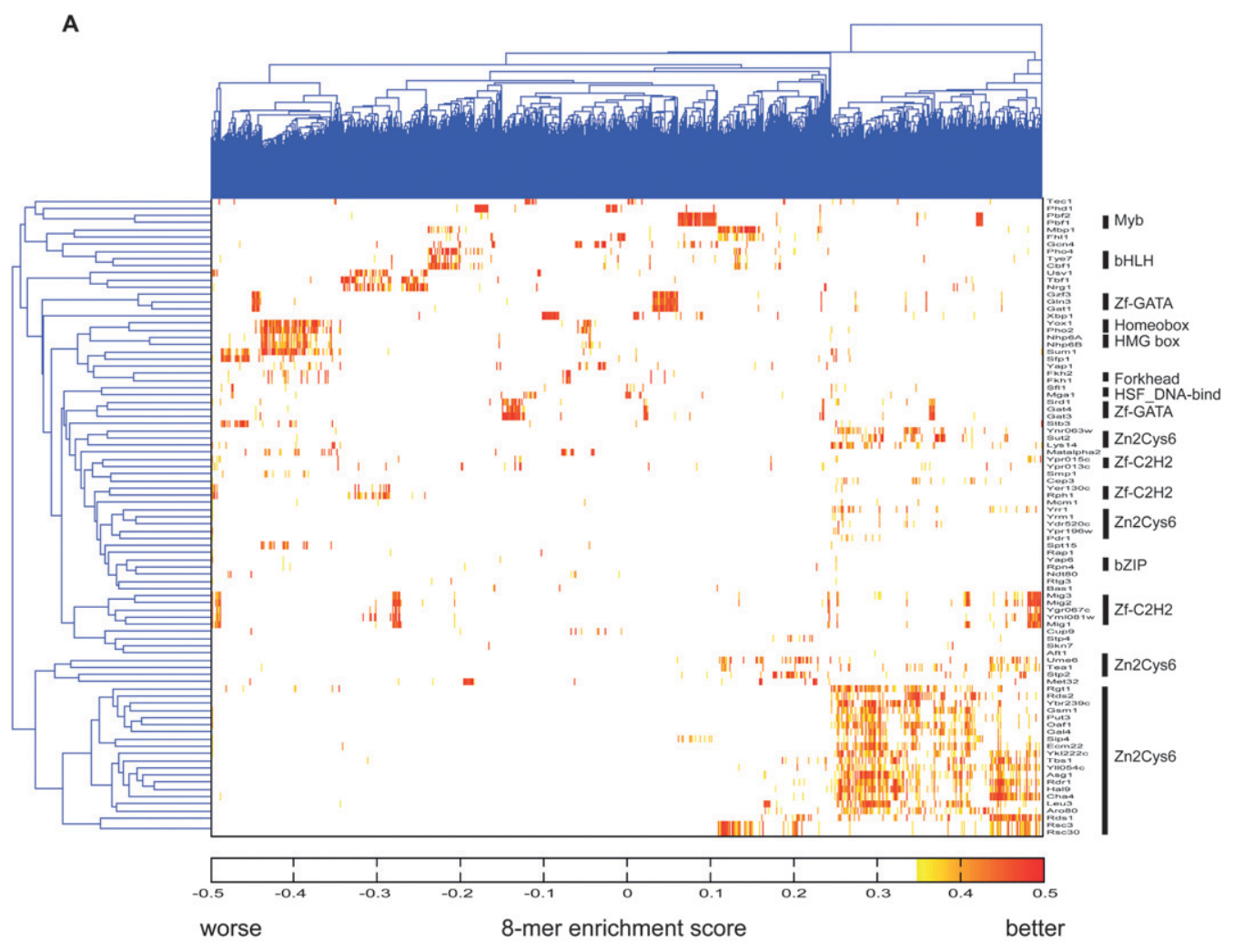

B

\begin{tabular}{|c|c|c|c|}
\hline TF & $\underline{\text { class }}$ & $\underline{\text { PBM }}$ & $\underline{\text { ChIP-chip motif }}$ \\
\hline Mcm1 & MADS & . . . & $\therefore C C$ A $I_{x} G_{A A A}$ \\
\hline Pho2 & homeobox & - s.TAATTA & AITAA \\
\hline Tec1 & TEA & ACATTCII & CATTC. \\
\hline Pbf1 & Myb & $=$ CGATGAs. & N/A \\
\hline Pbf2 & Myb & CGATGAG. & $\mathrm{N} / \mathrm{A}$ \\
\hline Stb3 & unknown & TGAAAAA & N/A \\
\hline Nhp6A & HMG & $\ldots \quad \ldots$ TATATAA & N/A \\
\hline Rsc30 & Zn2Cys6 & $\Rightarrow \mathbf{C C C G C G e s} \ldots \ldots$ & N/A \\
\hline Asg1 & Zn2Cys6 & $\ldots$ CCGG & N/A \\
\hline Hal9 & Zn2Cys6 & CGGA & N/A \\
\hline
\end{tabular}

Figure 1. PBM characterization of $S$. cerevisiae TF DNA-binding specificities. (A) Hierarchical clustering of PBM data over ungapped 8-mer E-scores determined for 89 yeast TFs. (B) Sequence logos for selected examples of newly discovered yeast TF DNA-binding site motifs.

Interestingly, we observed sequence-specific DNA-binding preferences by the non-histone chromatin proteins Nhp6A (Fig. 1B) and Nhp6B (Supplemental Fig. S4), neither of which previously had been thought to have sequence-specific DNA-binding activity (Giavara et al. 2005). We confirmed the specificities of a subset of these novel TFs for their PBM-derived DNA-binding sequences by electrophoretic mobility shift assays (EMSAs) (Supplemental Fig. S5).

We also report direct DNA-binding site motifs for 20 TFs for which our PBM-derived motifs are substantially different (CompareACE score < 0.7; Supplemental Table S2) from the motifs computationally inferred from ChIP-chip data (Harbison et al. 2004; Macisaac et al. 2006; Morozov and Siggia 2007) or from the literature, with our PBM-derived motifs being consistent with motifs of other TFs of the same DNA-binding domain structural classes. Three additional motifs are partial matches to previously derived motifs as the PBM motifs appear to be half-sites of apparently homodimeric ChIP-chip-derived motifs. Some of the ChIP-chip motifs that differ from PBM motifs appear to have captured a heterodimer. For example, the ChIP-chip-derived motif for Yox1 appears to contain not only a motif similar to our PBMderived Yox1 motif, but also a MADS domain motif; of note, Yox1 is known to interact with the MADS domain protein Mcm1 (Pramila et al. 2002). All together we report new, direct DNA-binding specificities for 50 known or candidate TFs and for eight proteins for which only a (matching) consensus sequence was previously known; in total, this corresponds to over a 50\% increase in the number of experimentally determined yeast DNA-binding site motifs (MacIsaac et al. 2006). Taking together our new PBM-derived 
motif data with prior motif data in the literature (MacIsaac et al. 2006; Morozov and Siggia 2007) or in the TRANSFAC database (Matys et al. 2003), experimentally determined DNA-binding site motif data are now available for 173 known or putative yeast TFs (Supplemental Table S3).

\section{Analysis of in vivo binding site data} with PBM k-mer data

Beyond looking at similarity between our PBM-derived motifs and literature motifs, we investigated the in vivo relevance of our $k$-mer data by comparing these data with ChIP-chip data. ChIP-chip experiments had previously been attempted for 70 of the 89 TFs in our data set as part of a large-scale survey (Harbison et al. 2004). We find that our $k$-mer binding data not only agree with the results of ChIP-chip for many TFs, but also aid in interpretation of the ChIP-chip data. Specifically, we used the $k$-mer binding data to calculate a predicted total TF occupancy score for each intergenic region (Supplemental Methods), ranked all of the intergenic regions by this score, and asked whether intergenic regions that scored well were enriched among the intergenic regions "bound" in vivo by ChIP-chip $(P<0.001)$ (Harbison et al. 2004).

For 33 of the 40 TFs for which we had both PBM- and ChIP-chip-derived motifs (Harbison et al. 2004), we observed good agreement (AUC $>0.5, P<$ 0.05) between the ChIP-chip in vivo data and our scoring of intergenic regions by the PBM $k$-mer data (Fig. 2A). For 20 of these TFs, scoring of intergenic regions by the $k$-mer data yielded better agreement with the ChIP-chip binding data (Harbison et al. 2004) than did scoring using the ChIP-chip-derived motifs (Fig. 2B; Supplemental Methods; Supplemental Fig. S6). In contrast, for all but five (Mig1, Smp1, Mga1, Rph1, Mig3) of the 17 TFs that did not yield ChIP-chip motifs (MacIsaac et al. 2006), the $k$-mer-derived potential target genes were not enriched within the ChIP-chip bound regions (Harbison et al. 2004) (AUC $>0.5, P<$ 0.05); this suggests that the direct targets of those 12 TFs were not enriched in those ChIP-chip data sets (Supplemental Fig. S6).

The high-resolution nature of the PBM data presented an opportunity to reanalyze the ChIP-chip data (Harbison et al. 2004) and the subsequent improved regulatory map published by MacIsaac et al. (2006) for how well the in vivo binding data fit a model of direct TF-DNA binding. Previous computational analyses of those ChIP-chip data were performed using computationally inferred PWMs learned from those same ChIP-chip data (Harbison et al. 2004; MacIsaac et al. 2006; Tanay 2006). In contrast, the PBM data were generated by a direct biochemical ap- proach, independent of the ChIP-chip experiments, and thus aid in annotation of direct targets.

We reanalyzed the individual targets identified as "bound" in vivo to investigate whether they are likely to be bound directly by the TFs or indirectly via interaction with other proteins. Previously, MacIsaac et al. (2006) identified a set of high-confidence binding sites, which they defined as those containing motif matches that were bound by the corresponding factor at $P<0.001$. We refer to those sites as that study's "direct" targets, and those bound by the corresponding factor at $P<0.001$, but not identified by MacIsaac et al. (2006) as containing a motif match as their "indirect" targets. We scanned each of the "bound" intergenic regions for the presence of at least one $k$-mer at $E>0.45$ (Berger et al. 2008), and annotated any such intergenic regions as "direct" targets according to $k$-mer matches and any bound intergenic 
regions not containing at least one $k$-mer at $E>0.45$ as "indirect" targets. Using an $E$-score threshold rather than a continuous occupancy score in this analysis provides a consistent way to determine sets of target intergenic regions across TFs for comparison with the discrete sets of target intergenic regions reported in the ChIP-chip results (Harbison et al. 2004). This resulted in our reassignment of 682 intergenic regions that previously had been classified as direct targets according to the MacIsaac et al. (2006) regulatory map, rather as potentially being indirect targets (Fig. 3). Moreover, this suggests that 653 bound intergenic regions that previously had not been classified as high-confidence target sites, according to MacIsaac and colleagues, may actually be direct targets for one of these 40 TFs (Fig. 3). Using this same approach to analyze an additional 17 TFs that had at least 10 bound intergenic regions $(P<0.001)$ (Harbison et al. 2004), but for which MacIsaac et al. (2006) had not derived motifs from the ChIP-chip data, we annotated 279 out of a total of 852 binding instances as direct targets at $E>0.45$ (Supplemental Table S4).

These comparisons highlight the complementary nature of the in vivo ChIP-chip and in vitro PBM data and the value of an integrated analysis for an improved distinction of direct versus indirect binding events. For example, the previously inferred ChIP-chip motifs for Fhl1 and Sfp1 (MacIsaac et al. 2006) appear to actually be matches to the Rap1 binding-site motif instead of reflecting their own respective DNA-binding specificities. Thus, some intergenic regions annotated by MacIsaac and colleagues as direct targets of Fhl1 or Sfp1 may actually be indirectly associated with those factors by interactions with Rap1. For other TFs, the large numbers of reannotated sites may be due to sensitivity to the PBM E-score threshold used. Since some TFs might utilize lower affinity DNA-binding sites than others, the use of different thresholds for different TFs may provide a more accurate distinction between direct and indirect binding sites; reannotation results at different $E$-score thresholds are shown in Supplemental Figure S7. For TFs for which relatively many intergenic regions previously annotated as indirect targets were reannotated as direct targets, the ChIP-chip-derived motif may not have accurately reflected the binding specificities of those factors (e.g., see earlier discussion of Yox1). In other cases, the PBM data suggest that a wider diversity of sequences may be recognized by a factor than is represented by the ChIP-chip motif, and thus, that more targets contain these additional preferred sequences.

\section{Effects of transcription factor perturbations on potential target genes}

To begin to investigate the potential regulatory functions of each $\mathrm{TF}$, we determined whether its top-ranked potential target genes, ranked according to the total occupancy score described above (Supplemental Methods; Supplemental Table S5), are over-represented for particular functional categories (Supplemental Table S6) (Tavazoie et al. 1999; Hughes et al. 2000a; Robinson et al. 2002). Function predictions using our $k$-mer binding data for previously characterized TFs generally were supported by the literature (Supplemental Fig. S8). However, DNA-binding specificity data alone cannot identify which particular binding-site sequence occurrences serve a regulatory role in vivo and which corresponding potential target genes are actually regulated by a TF.

Therefore, to further investigate the potential regulatory effects of these PBM-derived DNA-binding sites, we examined the effects of genetic perturbations (deletion, overexpression, or other gain-of-function or loss-of-function mutants) of the TFs on their PBM-derived potential target genes. For each gene expression microarray data set, we applied our CRACR algorithm (McCord et al. 2007) to determine whether potential target genes are significantly enriched among the up- or down-regulated genes in any of the gene expression arrays. In all, we considered 256 expression data sets for TF perturbation yeast strains that were available for 79 of the 89 TFs.

Significant effects of TF perturbations on their PBM-predicted target genes were found in many cases, and such support for the predicted target genes was found just as often for TFs with novel or significantly different motifs as for TFs with well-known motifs (Supplemental Table S7). Specifically, we found that the potential target genes of 44 of these 79 TFs were enriched at a conservative significance threshold (CRACR area statistic $\geq 0.095, P \leq 5 \times 10^{-4}$ ) for being either up- or down-regulated in a corresponding TF perturbation strain. Twenty of these 44 TFs correspond to those for which our PBM-derived motif is substantially different (CompareACE score < 0.7) from the motif computationally inferred (MacIsaac et al. 2006) from the ChIP-chip data (Harbison et al. 2004) or from the literature consensus sequence, and another 14 of these $44 \mathrm{TFs}$ correspond to those for which we newly report direct DNAbinding site motifs. For example, the potential target genes of Ypr015c, a putative protein of unknown function, are significantly enriched (CRACR area statistic $\left.=0.12, P=6.1 \times 10^{-10}\right)$ among genes down-regulated in a YPRO15C overexpression strain (Chua et al. 2006). More case examples for specific TFs are

\section{Genome Research}

www.genome.org 
provided in the following sections. The lack of enrichment for the other 35 TFs might indicate that those TFs did not exert a strong regulatory effect in the profiled conditions, that there exist at least partially functionally redundant TFs, or that the gene expression effects are predominantly due to secondary effects of the perturbed TFs. Support for the potential target genes of several additional TFs came from expression data on perturbations of genes with which they exhibit genetic interactions (Supplemental Table S7). Further experiments will be needed to validate our predictions for those cases where in vivo TF binding data and gene expression data on TF deletion or mutant TF strains are not available.

\section{Sequence-specific DNA binding by Rsc3 and Rsc30,} components of the RSC chromatin remodeling complex

RSC is an essential 15-subunit ATP-dependent chromatin remodeling complex that repositions nucleosomes (Cairns et al. 1996, 1999). Two subunits of this RSC complex, Rsc3 and Rsc30, are $\mathrm{Zn}_{2} \mathrm{Cys}_{6}$ proteins that previously had been hypothesized to recognize specific sequences and thus help target this important regulatory complex to specific genes (Wilson et al. 2006). In line with this hypothesis, we observed sequence-specific binding by these factors in PBM experiments and determined novel DNAbinding site motifs for them (Fig. 1B; Supplemental Fig. S4).

ChIP-chip data on in vivo occupancy by RSC support the model that in vivo genomic binding of the RSC complex is conferred by the Rsc3/30 sequence preferences that we have identified. ChIP-chip data are available for the Rsc1 and Rsc2 isoforms of the RSC complex from separate ChIP experiments on five subunits, namely, Rsc1, Rsc2, Rsc3, Rsc8, and Sth1 (Ng et al. 2002). In all five of these data sets, we found that the intergenic regions that scored well by the $k$-mer data for Rsc3, and separately for Rsc30, are highly enriched among intergenic regions occupied in vivo (Supplemental Table S8). The potential target regions of Rsc3 and Rsc30 are also enriched among the intergenic regions occupied by Rsc9 in four different environmental conditions (Damelin et al. 2002).

Furthermore, analysis of gene expression microarray data revealed that the potential target genes of Rsc3 and Rsc30 are enriched among the genes that are differentially expressed when Rsc3 or Rsc30 were perturbed. In a RSC3 temperature-sensitive mutant strain (Angus-Hill et al. 2001) in which RSC3 itself is upregulated, we found that Rsc3's potential target genes are enriched among down-regulated genes (CRACR area statistic $=0.110, P=$ $1.41 \times 10^{-6}$ ). In a RSC30 deletion strain (Angus-Hill et al. 2001), Rsc30's potential target genes are enriched among down-regulated genes $\left(\right.$ CRACR area statistic $\left.=0.099, P=1.70 \times 10^{-4}\right)$. Consistent with these findings, CRACR analysis of gene expression data performed on a RSC30 overexpressor strain (Chua et al. 2006), in which $R S C 3$ is up-regulated, revealed that the potential target genes of Rsc3 are enriched (CRACR area statistic $=0.0986$, $P=7.87 \times 10^{-4}$ ) among the repressed genes. These data support the model that Rsc3 and Rsc30 may sometimes have opposite functions (Angus-Hill et al. 2001).

Finally, Rsc3 and Rsc30 are required for regulation of genes that regulate cell wall integrity (Angus-Hill et al. 2001). Consistent with these findings, we observed that the PBM-derived potential target genes of Rsc3 are highly enriched for various functional annotation terms pertaining to the cell wall and cell wall function (Supplemental Table S6). In addition, Ng et al. (2002) found that RSC targets several gene classes, including histones. Consistent with that result, we found that Rsc3's PBM-derived potential target genes are also highly enriched for various functional categories of genes pertaining to chromatin remodeling (Supplemental Table S6). Taken together, all of these analyses support the model that Rsc3 and Rsc30 target the RSC complex to relevant classes of genes through the sequence preferences discovered in our PBM experiments.

\section{Two newly discovered PAC-binding factors associated with regulation of rRNA processing genes}

The PAC and RRPE motifs are highly over-represented in the upstream regions of rRNA processing and transcription genes (Hughes et al. 2000a), and exhibit significant correlation with their expression (Pilpel et al. 2001). While an RRPE-binding factor, Stb3, has recently been described (Liko et al. 2007), the identity of a PAC-binding factor had remained unknown. Two of our novel TFs' DNA-binding site motifs are extremely good matches to the well-known PAC motif (Dequard-Chablat et al. 1991), and we now refer to these proteins of previously unknown function as "PACbinding factor 1" (Pbf1; also known as Ybl054w) and "PAC-binding factor 2" (Pbf2; also known as Dot6, or Yer088c). By identifying Pbf 1 and Pbf 2 as PAC-binding factors, we have thus bridged a long-standing knowledge gap in the regulation of ribosome biogenesis. As expected given its recognition of the PAC motif, the genes predicted to have the highest occupancy by Pbf1 and Pbf2 are highly enriched for the Gene Ontology (GO) Biological Process terms "ribosome biogenesis" $\left(P=2.81 \times 10^{-8}\right.$ and $2.29 \times 10^{-7}$, respectively, Bonferroni-corrected $)$ and "rRNA processing" $(P=$ $8.28 \times 10^{-6}$ and $2.40 \times 10^{-3}$, respectively, Bonferroni-corrected) (Supplemental Table S6).

Experimental testing of these newly discovered TFs supports their involvement in regulation of rRNA processing genes. Consistent with prior studies of the association of the PAC motif with stress-induced changes in gene expression (Tavazoie et al. 1999; Causton et al. 2001; Pilpel et al. 2001), CRACR predicted that Pbf1 and Pbf 2 regulate rRNA processing genes in a number of stress conditions in which ribosome biosynthesis is repressed, including in heat shock (CRACR $P=4.9 \times 10^{-11}$ and $1.7 \times 10^{-11}$, respectively). Therefore, we performed Affymetrix gene expression profiling of the single deletion mutants $\Delta p b f 1$ and $\Delta p b f 2$, the double deletion mutant $\Delta p b f 1 \Delta p b f 2$, and the isogenic wild-type strain upon heat-shock treatment. We found that the potential target genes of Pbf1 and Pbf2 are significantly repressed (CRACR $P<10^{-12}$ ) during heat shock in wild-type and in the $\Delta p b f 1$ and $\Delta p b f 2$ single deletion strains, and that this repression is diminished in the $\Delta p b f 1 \Delta p b f 2$ double deletion strain (Fig. 4A; Supplemental S9). Consistent with the literature (Gasch et al. 2000), we found that during heat shock, rRNA processing genes are down-regulated (Supplemental Table S9). Importantly, we found that rRNA processing genes (GO ID 0006364) that contain at least one $k$-mer at a conservative threshold of $E \geq 0.45$ (Berger et al. 2008) for either Pbf 1 or Pbf 2 within 600 bp upstream of translational Start are significantly derepressed in $\Delta p b f 1\left(P=4.37 \times 10^{-13}\right.$, twotailed paired Wilcoxon-Mann-Whitney test) and in $\Delta p b f 2(P=3.19 \times$ $10^{-25}$, as above), and even more so in the $\Delta p b f 1 \Delta p b f 2$ double deletion strain $\left(P=4.41 \times 10^{-26}\right.$, as above) (Fig. 4B).

We used chromatin immunoprecipitation followed by quantitative PCR (ChIP-qPCR) to measure the association of Pbf1 and Pbf 2 under heat shock to the promoter regions of five rRNA processing genes-SAS10, NOP2, MTR4, KRR1, and ERBI-which contain the PAC motif, and as a negative control we used ENO2, which is not involved in rRNA processing and does not contain 
A

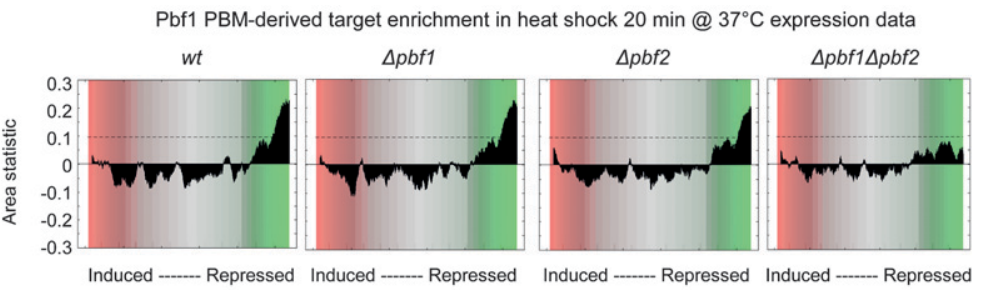

B

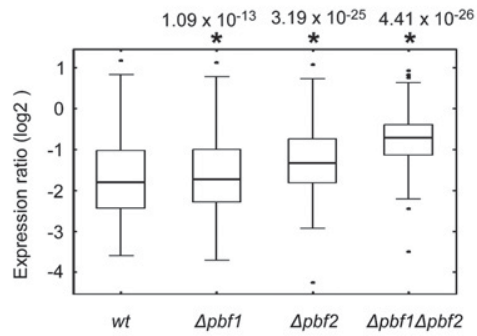

C
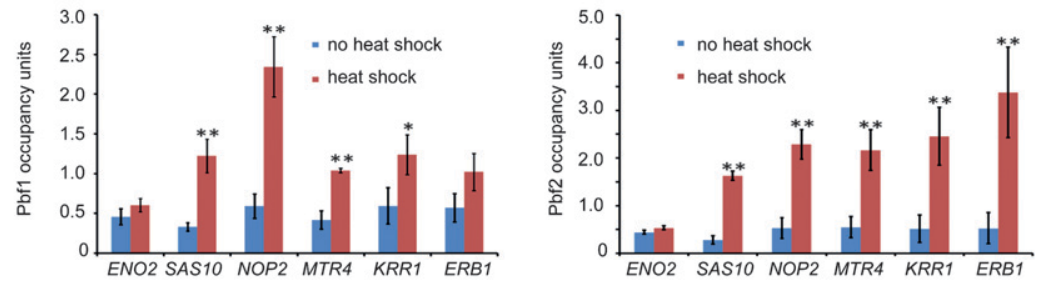

D

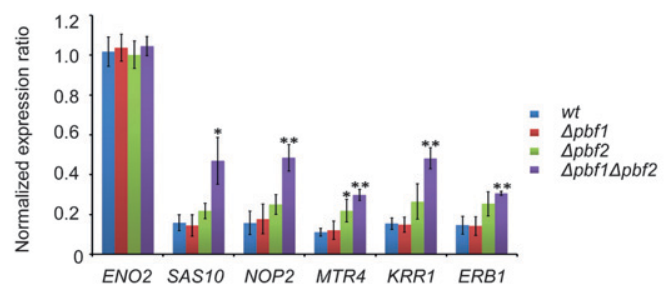

Figure 4. $\mathrm{Pbf} 1$ and $\mathrm{Pbf} 2$ regulate rRNA processing genes. (A) Predicted target genes of $\mathrm{Pbf} 1$ and $\mathrm{Pbf} 2$ are significantly repressed (CRACR $P<10^{-12}$ ) after 20 min heat shock (shift from $25^{\circ} \mathrm{C}$ to $37^{\circ} \mathrm{C}$ ) in wildtype, $\Delta p b f 1$, and $\Delta p b f 2$ strains, but not in the $\Delta p b f 1 \Delta p b f 2$ double deletion strain, in Affymetrix gene expression profiling of triplicate biological replicate cultures. (B) Box plots indicating expression changes of rRNA processing genes containing at least one $k$-mer at $E \geq 0.45$ after 20 min heat shock in wild-type, $\Delta p b f 1, \Delta p b f 2$, and $\Delta p b f 1 \Delta p b f 2$ strains, in the expression data from $A$. (C) Pbf1 and Pbf2 associate in vivo with the promoter regions of the rRNA processing genes SAS10, NOP2, MTR4, KRR1, and ERB1. ChIP-qPCR was performed on cells treated with 5-min heat shock, at predicted target sites in their upstream regions, and at a negative control region upstream of ENO2. Binding fold-enrichment was defined as the ratio of PCR product in "IP" versus "INPUT," using an open reading frame free region on chromosome $\mathrm{V}$ as an internal normalization control. Error bars indicate 1 SD from triplicate biological replicate cultures $\left({ }^{*} P<0.05 ;{ }^{* *} P<0.01\right.$; two-sided Student's $t$-test). $(D)$ Expression ratio of rRNA processing genes after heat shock. RT-qPCR data were generated for either untreated yeast or yeast treated with 20-min heat shock. Gene expression was normalized relative to $A C T 1$ as an internal normalization control. Error bars indicate 1 SD from triplicate biological replicate cultures $\left({ }^{\star} P<0.05\right.$; ${ }^{* *} P<0.01$; two-sided Student's $t$-test compared with wild type).

a PAC site in its promoter region. We found that after heat shock, Pbf 2 bound significantly ( $P<0.01$, Student's $t$-test) to all five of these rRNA processing genes' promoters, and not to ENO2 (Fig. 4C). Similarly, Pbf1 bound significantly to most of these promoters, although to a lesser degree. Quantitative RT-PCR data indicated that all five of these rRNA processing genes are normally repressed during heat shock, but that they are all significantly derepressed $(P<0.05$, Student's $t$-test) in the $\Delta p b f 1 \Delta p b f 2$ double deletion strain (Fig. 4D). The derepression appeared to be greater in $\Delta p b f 2$ than in $\Delta p b f 1$, but the derepression was statistically significant for only one of these five genes in the $\Delta p b f 2$ single de- letion mutant. Taking all of these data together, we conclude that both Pbf1 and Pbf 2 coregulate rRNA processing genes upon heat shock, with Pbf2 apparently playing a greater role.

\section{Prediction of transcription factor condition-specific regulatory roles}

Condition-specific binding-site usage is a vital aspect of TF function (Simon et al. 2001; Harbison et al. 2004). While ChIPchip provides a "snapshot" of what genomic regions are occupied in vivo by a TF either directly or indirectly in the particular examined condition(s), PBM data provide information on the inherent, direct DNA-binding preferences of a protein. Therefore, we applied our CRACR algorithm (McCord et al. 2007) to the potential target genes of these $89 \mathrm{~S}$. cerevisiae TFs in order to generate specific hypotheses about the condition-specific binding-site utilization and functions of these TFs, considering the environmental and cellular conditions represented in 1693 publicly available microarray gene expression data sets (Supplemental Tables S10, S11). Some of these CRACR predictions have been experimentally validated. Using ChIP-qPCR, we validated our CRACR predictions of conditionspecific Rap1 binding site usage after diamide treatment (Supplemental Fig. S10). In addition, CRACR predicted that Pbf1 and $\mathrm{Pbf} 2$ coregulate rRNA processing genes in heat shock (Fig. 4; Supplemental Table S10).

The CRACR results from analysis of 1693 conditions suggested conditionspecific regulation by additional previously uncharacterized TFs in conditions including sporulation, carbohydrate metabolism, and stress. The predicted conditions could be used to direct future in vivo experiments in cases where previous experiments may have failed because the yeast were grown in conditions in which the TF of interest does not bind its target sites. For example, a prior Tbs1 ChIP-chip experiment (Harbison et al. 2004) was performed on yeast grown only in YPD rich media conditions in the absence of stress, while CRACR suggests that Tbs1 regulates its target genes in sodium chloride and heat-shock stress conditions.

\section{Identification of potential coregulatory transcription factors}

To identify sets of TFs that may exhibit similar regulatory effects on their target genes over various conditions, we performed twodimensional hierarchical clustering of the 89 TFs according to their CRACR statistics across all 1693 microarray expression data sets (Fig. 5A; Supplemental Fig. S11). This CRACR clustering analysis

\section{Genome Research www.genome.org}




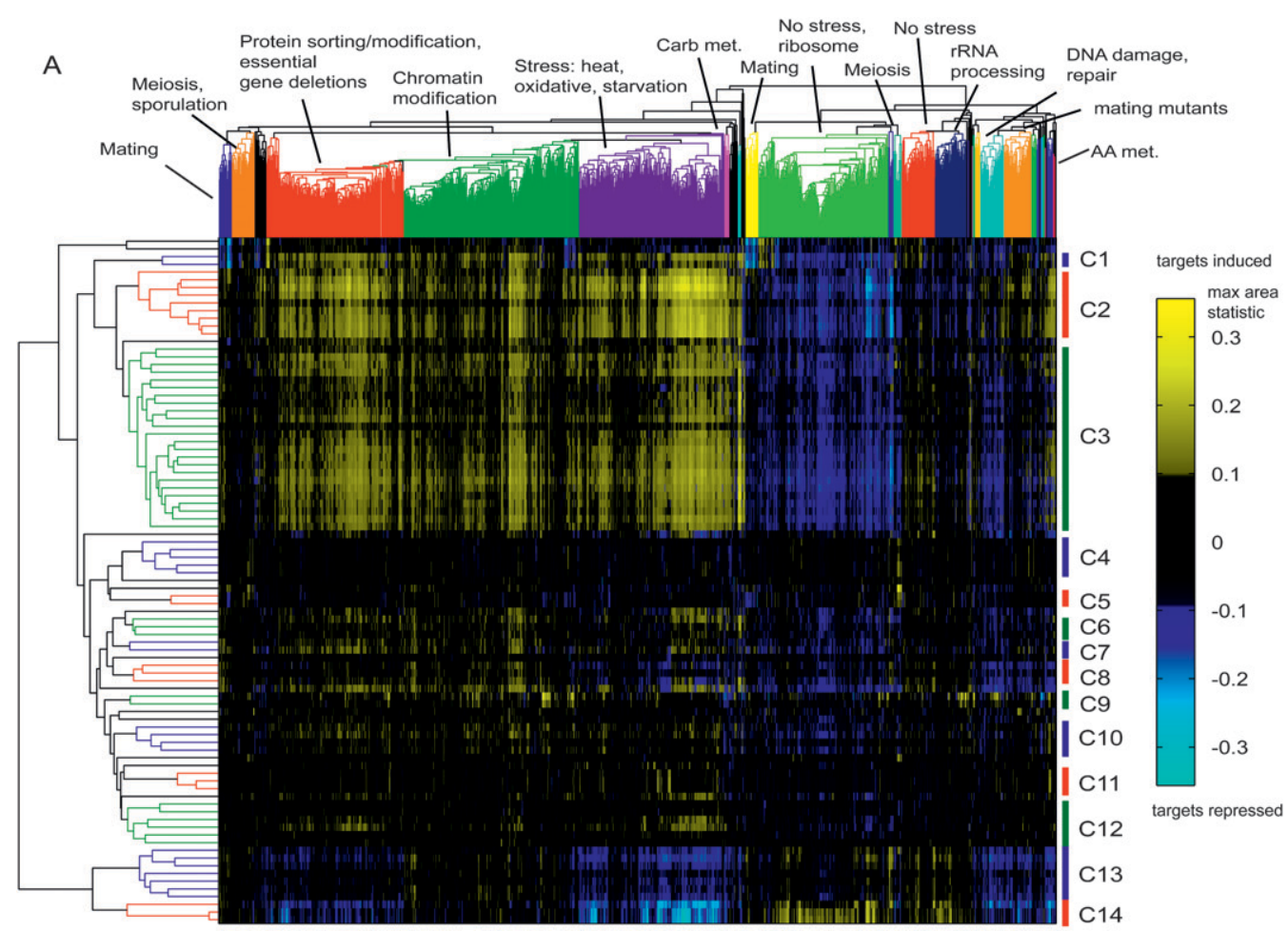

B

\begin{tabular}{|c|c|c|c|c|}
\hline Cluster & Function & & Selected Motifs & Selected k-mer Correlations \\
\hline C2 & $\begin{array}{l}\text { Carbohydrate } \\
\text { metabolism, } \\
\text { response to stress }\end{array}$ & $\begin{array}{l}\text { Mig1: } \\
\text { Rph1: }\end{array}$ & $\begin{array}{l}\text { AATCGGGG. } \\
\text { مITAGGGG. }\end{array}$ & $\begin{array}{l}\text { Mig1/Mig2: } 0.870 \\
\text { Mig2/Rph1: } 0.0924 \\
\text { Mig3/Nrg1: } 0.078\end{array}$ \\
\hline C3 & $\begin{array}{l}\text { Stress response, } \\
\text { drug response }\end{array}$ & $\begin{array}{l}\text { Ecm22: } \\
\text { Ume6: } \\
\text { Ynro63w: } \\
\text { Yrr1: }\end{array}$ & 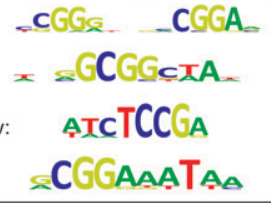 & $\begin{array}{l}\text { Ume6/Yrr1: }-0.0259 \\
\text { Ume6/Ynr063w: } 0.0324 \\
\text { Stp2/Ynr063w: }-0.082 \\
\text { Rds1/Yrr1: } 0.035\end{array}$ \\
\hline C8 & $\begin{array}{l}\text { Transport (carb. } \\
\text { etc) }\end{array}$ & $\begin{array}{l}\text { Lys14: } \\
\text { Sfl1: }\end{array}$ & $\begin{array}{l}\text { AAATTCCG } \\
\text { CTTC }\end{array}$ & $\begin{array}{l}\text { Lys14/Sfl1: } 0.376 \\
\text { Ypr196w/Lys14: } 0.367 \\
\text { Sfl1/Ypr196w: - }-0.008\end{array}$ \\
\hline C10 & $\begin{array}{l}\text { AA and } \\
\text { phosphate } \\
\text { metabolism }\end{array}$ & $\begin{array}{l}\text { Met32: } \\
\text { Tye7: }\end{array}$ & $\begin{array}{l}\text { TATGGCG } \\
\text { =AICACGTGAI }\end{array}$ & $\begin{array}{l}\text { Tye7/Cbf1: } 0.6348 \\
\text { Cbf1/Pho4: } 0.629 \\
\text { Met32/Tye7: } 0.038\end{array}$ \\
\hline C11 & $\begin{array}{l}\text { Nitrogen and } \\
\text { sulfur metabolism }\end{array}$ & $\begin{array}{l}\text { Gln3: } \\
\text { Gat1: }\end{array}$ & $\begin{array}{c}\text { s_TTATC } \\
\text { cTTATC }\end{array}$ & $\begin{array}{l}\text { Gzf3/Gln3: } 0.874 \\
\text { Gzf3/Gat1: } 0.951 \\
\text { Gln3/Gat1: } 0.901\end{array}$ \\
\hline C14 & $\begin{array}{l}\text { rRNA processing, } \\
\text { ribososome }\end{array}$ & $\begin{array}{l}\text { Pbf2: } \\
\text { Stb3: }\end{array}$ & $\begin{array}{l}\text { CGATGAG }= \\
\text { AsTTTTICA.- }\end{array}$ & $\begin{array}{l}\text { Pbf1/Pbf2: } 0.928 \\
\text { Pbf2/Stb3: }-0.040 \\
\text { Pbf1/Stb3: }-0.048\end{array}$ \\
\hline
\end{tabular}

Figure 5. Analysis of TFs' regulatory associations and coregulatory factors. (A) Two-dimensional hierarchical clustering of 89 TFs (rows) according to their CRACR statistics across 1693 expression conditions (columns). (B) Examples of predicted coregulatory TFs from $A$ with distinct motifs, and their 8mer binding profile correlations. Clusters annotations are derived from the literature and functional predictions from this study. A high-resolution heatmap with full labeling is available in Supplemental Fig. S11, S12.

provides different information from clustering the TFs' own gene expression patterns, as it identifies the conditions in which the TFs' potential target genes are differentially expressed rather than when the TFs themselves are differentially expressed. This clustering analysis also provides different information than an analysis of overlap in the sets of intergenic regions bound in ChIP-chip (Harbison et al. 2004), where binding events could involve either direct DNA binding or indirect DNA associations by the TFs.
Expression conditions in general segregated according to general functional categories, and various sets of TFs have similar regulatory associations across conditions. The largest pattern in the CRACR clustering heatmap includes numerous TFs generally involved in stress response that appear to regulate their genes in response to various stress conditions. Using Pbf1, Pbf2, and Stb3 as a guide because of their DNA-binding site motifs' known coregulatory associations (Hughes et al. 2000a; Pilpel et al. 2001; Beer 
and Tavazoie 2004), we considered all TF clusters with equal or greater CRACR profile similarity as that of the Pbf1/Pbf $2 / \mathrm{Stb} 3$ cluster, as groups of putative coregulatory TFs (Fig. 5B; Supplemental Fig. S12). At this threshold, 14 of 18 TFs predicted to be involved in drug response according to over-represented annotation terms among their potential target genes (Supplemental Table S6) clustered together in cluster C3 (Supplemental Fig. S12); these include the novel candidate drug response TFs Asg1 and Ykl222c as well as the known drug response TF Yrr1. While some clusters, such as C11, identified coregulatory TFs that have very similar $k$-mer binding profiles, other clusters identified coregulatory TFs with distinct motifs (Fig. 5B; Supplemental Fig. S12). Such hypotheses about potential coregulation can be used to direct future in vivo experimentation on yeast gene regulatory networks.

\section{Discussion}

This study provides both comprehensive binding specificity data for many known and newly identified TFs and a framework for identifying all yeast DNA-binding proteins and predicting their regulatory roles. The use of experimentally determined, comprehensive $k$-mer binding data to identify candidate cis regulatory elements and to predict candidate target genes is novel not only in the depth of experimentally measured TF binding specificity data, but also in tactical approaches for scoring genomic sequence for its regulatory potential. We expect that these yeast data will serve as a valuable model system for developing new, $k$-mer-based approaches for modeling transcriptional regulatory networks. Since PBM data correlate with DNA-binding affinities (Berger et al. 2006), in the future, the methods and data presented here could be adapted to explore differences in the usage of binding sites of different affinities between TFs or in different cellular or environmental conditions. Finally, although many challenges exist in the identification of cis regulatory elements in higher eukaryotes because of their frequently more distant regulatory regions and greater combinatorial regulatory mechanisms, the approaches described in this study could be integrated with additional strategies, such as phylogenetic footprinting, nucleosome occupancy data, high-resolution ChIP-chip, or ChIP-seq, in order to identify trans regulatory factors, to predict their cis regulatory elements and target genes, and to distinguish their directly bound and indirectly associated regulatory sites in genomes.

\section{Methods}

\section{Cloning, expression, and purification of $S$. cerevisiae TFs}

We cloned the 245 full-length ORFs and 99 DBDs into Gatewaycompatible Entry and Destination vectors, pDONR201 or pDONR221, and pDEST-GST (Braun et al. 2002), as described previously (Hu et al. 2007). A separate, partially-redundant set of 118 DBDs were a generous gift from Tim Hughes (University of Toronto, Ontario, Canada) and Jason Lieb (University of North Carolina, Chapel Hill). Thus, our final clone collection includes 245 ORFs as full-length constructs, and 208 as DBDs. We did not pursue cloning of DBDs if the Pfam-annotated DBDs spanned $>80 \%$ of the full-length protein, or in some cases, if careful manual inspection indicated that these were not likely DBDs based on their descriptions (e.g., bromodomain).

\section{Protein production}

For all 245 full-length TF and 208 DBD clones, we performed highthroughput overexpression in E. coli cultures and subsequent af- finity purification using glutathione resin in 96-well plates, essentially as described previously (Hu et al. 2007). For each purified protein we performed Western blots to assess quality and to approximate its concentration. Overall, this resulted in 246 nonredundant TFs.

\section{Protein-binding microarrays (PBMs)}

We constructed microarrays covering all 10-bp sequence variants (Berger et al. 2006; Philippakis et al. 2008) by converting highdensity single-stranded oligonucleotide arrays to double-stranded DNA arrays (Berger et al. 2006; Berger and Bulyk 2009). Using these universal arrays, we measured the relative preferences of a TF for all possible contiguous 8-mers, as well as gapped 8-mers spanning up to 10 total positions. The new array designs we developed for this study also included typically 32 -fold redundancy for all non-palindromic 8-mers consisting of two 4-bp half-sites separated by spacers from 1 to $12 \mathrm{bp}$ in length; we added this array design feature to allow us to capture the sequence preferences of TFs with long or gapped recognition motifs, such as members of the $\mathrm{Zn}_{2} \mathrm{Cys}_{6}$ structural class (i.e., "Gal4-type" motifs).

\section{Identification of DNA-binding specificities}

Every nonpalindromic 8-mer occurs on at least 32 spots in each chamber of our universal PBM. Because of this redundancy, we are able to provide a robust estimate of the relative preference of a TF for every contiguous and gapped 8-mer that is covered on our array. Here, for each 8-mer, we provide the median normalized signal intensity and a rank-based statistical enrichment score (E-score). Median normalized signal intensity refers to the median normalized signal intensity for the set of probes containing a match to each 8-mer (usually $\sim 32$ probes). Our $E$-score is a rankbased, nonparametric statistical measure that has been described previously in detail (Berger et al. 2006) and ranges from +0.5 (most favored) to -0.5 (most disfavored). We applied our "Seed-andWobble" algorithm to derive position weight matrices (PWMs) from universal array PBM data (Berger et al. 2006; Berger and Bulyk 2009). All PBM data are publicly available via the UniPROBE database (Newburger and Bulyk 2009).

\section{Comparison of PBM motifs}

We used CompareACE (Hughes et al. 2000a) to compare our 89 PBM-derived motifs against a list of 4282 PWMs for previously published motifs. We required a minimum CompareACE motif similarity score of 0.7 to consider motifs as matching.

\section{Identification and scoring of potential target genes using PBM $k$-mer data}

A predicted total occupancy score for a given TF was calculated for the upstream promoter region of each gene by summing the background-subtracted median PBM signal intensities for each overlapping 8-mer, considering all those 8-mers at $E \geq 0.35$, over the sequence up to $600 \mathrm{bp}$ upstream of translation start. The median value of the median intensities over all 8-mers was used as a measure of the background signal and was subtracted from each individual 8-mer's intensity before summation. For most analyses, the total occupancy score was used to rank genes according to their likelihood of being TF target genes. The top 200 scoring genes were considered for analysis of functional category enrichment among a TF's potential target genes. We utilized our CRACR algorithm essentially as described previously (McCord et al. 2007), except here, genes were first ranked by the predicted total occupancy

\section{Genome Research} www.genome.org 
of the sequence up to $600 \mathrm{bp}$ upstream of its translational start site by a TF as described above.

\section{Analysis of ChIP-chip data}

All yeast intergenic regions (IGRs) were scored using PBM 8-mer data as described above for each TF, except that in this case the entire IGR length was scored, rather than only 600 bp upstream of translation start. This enabled a direct comparison between scores derived from PBM data and those measured from ChIP-chip experiments, in which the entire IGR was included on the array. Target IGR sets for each TF were defined as IGRs bound by the TF in a ChIP-chip experiment at $P<0.001$ in any experimental condition, as reported by the authors of that study (Harbison et al. 2004). TFs were excluded from the analysis if fewer than 10 IGRs were bound at $P<0.001$ in the ChIP-chip data. An area under the receiver operating characteristic (ROC) curve (AUC statistic) was then calculated by comparing the PBM-derived ranks of IGRs within the ChIP-chip "bound" IGRs (foreground set, or "class 1") to the ranks of the rest of the yeast IGRs (background set, or "class 0 "). For comparison, ChIP-chip-derived motifs, if available, were used to rank the IGRs as well. ScanACE (Roth et al. 1998; Hughes et al. 2000b) was used to score ChIP-chip motif matches in all yeast IGRs at a threshold of $2 \mathrm{SD}$ below the mean motif score. If multiple matches occurred within an IGR, these scores were summed to obtain a final score for each IGR. The resulting ChIP-chip IGR ranking was then used to calculate an AUC statistic comparing the ChIP-chip-derived ranks for ChIP-chip target IGRs versus background IGRs.

\section{EMSAs}

Sixty-nucleotide EMSA probes were designed such that the $5^{\prime} 40$ nt sequence corresponds to a putative target intergenic region in the yeast genome and contains the predicted DNA-binding site, and the next $20 \mathrm{nt}$ corresponds to a common priming sequence at the $3^{\prime}$ end that can anneal to a universal biotinylated primer. Primer extensions reactions were performed in order to convert the single-stranded probes to double-stranded probes. Approximately $5 \mathrm{nM}$ DNA probe and $\sim 0.2 \mu \mathrm{M}$ protein were used in each reaction.

\section{Yeast strains and growth conditions}

$B Y 4741, \Delta p b f 1$, and $\Delta p b f 2$ were purchased from Open Biosystems. The $\Delta p b f 1 \Delta p b f 2$ double deletion mutant was generated by replacing PBF2 with URA3 by homologous recombination in the $\triangle p b f 1$ background. PCR epitope tagging was used to generate yeast strains with a 3xHA (hemagglutinin) $\mathrm{N}$-terminal epitope tag using plasmid pMPY-3xHA. All yeast were grown in standard yeast YPD medium if not otherwise specified.

\section{Chromatin immunoprecipitation (ChIP) and quantitative PCR (qPCR)}

We carried out chromatin immunoprecipitation as described previously (Aparicio et al. 2005) with minor modifications. Three independent cultures were grown in parallel in order to carry out triplicate biological replicates for ChIP assays. Cells were then subjected to heat-shock treatment, i.e., growth temperature shifted from $25^{\circ} \mathrm{C}$ to $37^{\circ} \mathrm{C}$, for $5 \mathrm{~min}$, prior to fixation with $1 \%$ formaldehyde for $20 \mathrm{~min}$. qPCRs were performed using iQ SYBR Green SuperMix (Bio-Rad) on an iCycler real-time PCR thermocycler.

\section{Gene expression profiling and quantitative RT-PCR (RT-qPCR)}

Three independent cultures of the BY4741, $\Delta p b f 1, \Delta p b f 2$, and $\Delta p b f 1 \Delta p b f 2$ strains were grown in parallel in order to carry out triplicate biological replicates. Cells were then subjected to heatshock treatment, i.e., growth temperature shifted from $25^{\circ} \mathrm{C}$ to $37^{\circ} \mathrm{C}$, for $20 \mathrm{~min}$, and subsequently spun down and flash-frozen at $-80^{\circ} \mathrm{C}$. RNA was extracted and purified using Qiagen RNeasy Mini kit with DNase I treatment. Gene expression profiling was performed using Affymetrix Yeast Genome 2.0 GeneChip oligonucleotide arrays essentially according to Affymetrix protocols. Microarray data were analyzed as described previously (Choe et al. 2005). We imposed a false discovery rate (FDR) of 0.0001 as the cut-off value to identify differentially expressed genes. Microarray data were deposited into the GEO database under accession number GSE13684. GO term enrichment analysis was performed by applying FuncAssociate (Berriz et al. 2003) on lists of differentially expressed genes ordered by their expression ratio. RT-qPCR reactions were performed essentially as described above.

\section{Acknowledgments}

We thank G. Badis, T.R. Hughes, and J.D. Lieb for sharing 118 N-term GST fusion DNA-binding domain expression clones; G. Berriz for assistance with FuncAssociate; F. Winston, I. Laprade, and $\mathrm{X}$. Li for technical assistance with yeast experiments; J.-A. Kwon and J. Love for technical assistance with Affymetrix gene expression profiling experiments; T. Gurbich for technical assistance with PBMs; and K. Struhl for helpful discussion. Plasmid pMPY-3xHA was a generous gift from K. Struhl. We thank S. Gisselbrecht, T. Siggers, and A. Dudley for critical reading of the manuscript. We also thank anonymous reviewers for helpful comments. This work was funded by grants R01 HG003985 and R01 HG003420 from NIH/NHGRI to M.L.B. M.F.B. and R.P.M. were supported in part by National Science Foundation Graduate Research Fellowships.

\section{References}

Angus-Hill, M.L., Schlichter, A., Roberts, D., Erdjument-Bromage, H., Tempst, P., and Cairns, B.R. 2001. A Rsc3/Rsc30 zinc cluster dimer reveals novel roles for the chromatin remodeler RSC in gene expression and cell cycle control. Mol. Cell 7: 741-751.

Aparicio, O., Geisberg, J., Sekinger, E., Yang, A., Moqtaderi, Z., and Struhl, K. 2005. Chromatin immunoprecipitation for determining the association of proteins with specific genomic sequences in vivo. Curr. Protoc. Mol. Biol. doi: 10.1002/0471142727.mb2103s69.

Beer, M.A. and Tavazoie, S. 2004. Predicting gene expression from sequence. Cell 117: 185-198.

Berger, M. and Bulyk, M. 2009. Universal protein binding microarrays for the comprehensive characterization of the DNA binding specificities of transcription factors. Nat. Protocols. (in press).

Berger, M.F., Philippakis, A.A., Qureshi, A.M., He, F.S., Estep III., P.W., and Bulyk, M.L. 2006. Compact, universal DNA microarrays to comprehensively determine transcription-factor binding site specificities. Nat. Biotechnol. 24: 1429-1435.

Berger, M.F., Badis, G., Gehrke, A.R., Talukder, S., Philippakis, A.A., PenaCastillo, L., Alleyne, T.M., Mnaimneh, S., Botvinnik, O.B., Chan, E.T., et al. 2008. Variation in homeodomain DNA binding revealed by highresolution analysis of sequence preferences. Cell 133: 1266-1276.

Berriz, G.F., King, O.D., Bryant, B., Sander, C., and Roth, F.P. 2003. Characterizing gene sets with FuncAssociate. Bioinformatics 19: 25022504.

Borneman, A.R., Zhang, Z.D., Rozowsky, J., Seringhaus, M.R., Gerstein, M., and Snyder, M. 2007. Transcription factor binding site identification in yeast: A comparison of high-density oligonucleotide and PCR-based microarray platforms. Funct. Integr. Genomics 7: 335-345.

Braun, P., Hu, Y., Shen, B., Halleck, A., Koundinya, M., Harlow, E., and LaBaer, J. 2002. Proteome-scale purification of human proteins from bacteria. Proc. Natl. Acad. Sci. 99: 2654-2659.

Bulyk, M.L. 2006. DNA microarray technologies for measuring proteinDNA interactions. Curr. Opin. Biotechnol. 17: 422-430. 
Bulyk, M.L., Gentalen, E., Lockhart, D.J., and Church, G.M. 1999. Quantifying DNA-protein interactions by double-stranded DNA arrays. Nat. Biotechnol. 17: 573-577.

Bulyk, M.L., Huang, X., Choo, Y., and Church, G.M. 2001. Exploring the DNA-binding specificities of zinc fingers with DNA microarrays. Proc. Natl. Acad. Sci. 98: 7158-7163.

Cairns, B.R., Lorch, Y., Li, Y., Zhang, M., Lacomis, L., Erdjument-Bromage, H., Tempst, P., Du, J., Laurent, B., and Kornberg, R.D. 1996. RSC, an essential, abundant chromatin-remodeling complex. Cell 87: 1249_ 1260 .

Cairns, B.R., Schlichter, A., Erdjument-Bromage, H., Tempst, P., Kornberg, R.D., and Winston, F. 1999. Two functionally distinct forms of the RSC nucleosome-remodeling complex, containing essential AT hook, BAH, and bromodomains. Mol. Cell 4: 715-723.

Causton, H.C., Ren, B., Koh, S.S., Harbison, C.T., Kanin, E., Jennings, E.G., Lee, T.I., True, H.L., Lander, E.S., and Young, R.A. 2001. Remodeling of yeast genome expression in response to environmental changes. Mol. Biol. Cell 12: 323-337.

Choe, S.E., Boutros, M., Michelson, A.M., Church, G.M., and Halfon, M.S 2005. Preferred analysis methods for Affymetrix GeneChips revealed by a wholly defined control dataset. Genome Biol. 6: R16. doi: 10.1186/gb2005-6-r16.

Chua, G., Morris, Q.D., Sopko, R., Robinson, M.D., Ryan, O., Chan, E.T., Frey, B.J., Andrews, B.J., Boone, C., and Hughes, T.R. 2006. Identifying transcription factor functions and targets by phenotypic activation. Proc. Natl. Acad. Sci. 103: 12045-12050.

Cliften, P., Sudarsanam, P., Desikan, A., Fulton, L., Fulton, B., Majors, J., Waterston, R., Cohen, B., and Johnston, M. 2003. Finding functional features in Saccharomyces genomes by phylogenetic footprinting. Science 301: $71-76$

Damelin, M., Simon, I., Moy, T.I., Wilson, B., Komili, S., Tempst, P., Roth, F.P. Young, R.A. Cairns, B.R., and Silver, P.A. 2002. The genome-wide localization of Rsc9, a component of the RSC chromatin-remodeling complex, changes in response to stress. Mol. Cell 9: 563-573.

Dequard-Chablat, M., Riva, M., Carles, C., and Sentenac, A. 1991. RPC19, the gene for a subunit common to yeast RNA polymerases A (I) and C (III). J. Biol. Chem. 266: 15300-15307.

Gasch, A., Spellman, P., Kao, C., Carmel-Harel, O., Eisen, M., Storz, G., Botstein, D., and Brown, P. 2000. Genomic expression programs in the response of yeast cells to environmental changes. Mol. Biol. Cell 11: 4241-4257.

Gasch, A.P., Moses, A.M., Chiang, D.Y., Fraser, H.B., Berardini, M., and Eisen, M.B. 2004. Conservation and evolution of cis-regulatory systems in ascomycete fungi. PLoS Biol. 2: e398. doi: 10.1371/journal.pbio. 0020398 .

Giavara, S., Kosmidou, E., Hande, M.P., Bianchi, M.E., Morgan, A., d'Adda di Fagagna, F, and Jackson, S.P 2005. Yeast Nhp6A/B and mammalian Hmgb1 facilitate the maintenance of genome stability. Curr. Biol. 15: 68-72.

Harbison, C.T., Gordon, D.B., Lee, T.I., Rinaldi, N.J., Macisaac, K.D., Danford, T.W., Hannett, N.M., Tagne, J.B., Reynolds, D.B., Yoo, J., et al 2004. Transcriptional regulatory code of a eukaryotic genome. Nature 431: 99-104.

Hu, Y., Rolfs, A., Bhullar, B., Murthy, T., Zhu, C., Berger, M., Camargo, A., Kelley, F., McCarron, S., Jepson, D., et al. 2007. Approaching a complete repository of sequence-verified, protein-encoding clones for Saccharomyces cerevisiae. Genome Res. 17: 536-543.

Hughes, J.D., Estep, P.W., Tavazoie, S., and Church, G.M. 2000a. Computational identification of cis-regulatory elements associated with groups of functionally related genes in Saccharomyces cerevisiae. J. Mol. Biol. 296: 1205-1214.

Hughes, T., Marton, M., Jones, A., Roberts, C., Stoughton, R., Armour, C., Bennett, H., Coffey, E., Dai, H., He, Y., et al. 2000b. Functional discovery via a compendium of expression profiles. Cell 102: 109-126.

Ideker, T., Thorsson, V., Ranish, J.A., Christmas, R., Buhler, J., Eng, J.K., Bumgarner, R., Goodlett, D.R., Aebersold, R., and Hood, L. 2001 Integrated genomic and proteomic analyses of a systematically perturbed metabolic network. Science 292: 929-934.

Iyer, V.R., Horak, C.E., Scafe, C.S., Botstein, D., Snyder, M., and Brown, P.O. 2001. Genomic binding sites of the yeast cell-cycle transcription factors SBF and MBF. Nature 409: 533-538.

Johnson, D.S., Mortazavi, A., Myers, R.M., and Wold, B. 2007. Genomewide mapping of in vivo protein-DNA interactions. Science 316: 1497 1502.

Kellis, M., Patterson, N., Endrizzi, M., Birren, B., and Lander, E. 2003. Sequencing and comparison of yeast species to identify genes and regulatory elements. Nature 423: 241-254.

Liang, S.D., Marmorstein, R., Harrison, S.C., and Ptashne, M. 1996. DNA sequence preferences of GAL4 and PPR1: How a subset of $\mathrm{Zn}_{2} \mathrm{Cys}_{6}$ binuclear cluster proteins recognizes DNA. Mol. Cell. Biol. 16: 37733780 .
Lieb, J.D., Liu, X., Botstein, D., and Brown, P.O. 2001. Promoter-specific binding of Rap1 revealed by genome-wide maps of protein-DNA association. Nat. Genet. 28: 327-334.

Liko, D., Slattery, M.G., and Heideman, W. 2007. Stb3 binds to ribosomal RNA processing element motifs that control transcriptional responses to growth in Saccharomyces cerevisiae. J. Biol. Chem. 282: 26623-26628.

MacIsaac, K.D., Wang, T., Gordon, D.B., Gifford, D.K., Stormo, G.D., and Fraenkel, E. 2006. An improved map of conserved regulatory sites for Saccharomyces cerevisiae. BMC Bioinformatics 7: 113. doi: 10.1186/14712105-7-113

Mamane, Y., Hellauer, K., Rochon, M.H., and Turcotte, B. 1998. A linker region of the yeast zinc cluster protein leu3p specifies binding to everted repeat DNA. J. Biol. Chem. 273: 18556-18561.

Matys, V., Fricke, E., Geffers, R., Gossling, E., Haubrock, M., Hehl, R., Hornischer, K., Karas, D., Kel, A.E., Kel-Margoulis, O.V., et al. 2003. TRANSFAC: Transcriptional regulation, from patterns to profiles. Nucleic Acids Res. 31: 374-378.

McCord, R.P., Berger, M.F., Philippakis, A.A., and Bulyk, M.L. 2007. Inferring condition-specific transcription factor function from DNA binding and gene expression data. Mol. Syst. Biol. 3: 100. doi: 10.1038/msb4100140.

Morozov, A.V. and Siggia, E.D. 2007. Connecting protein structure with predictions of regulatory sites. Proc. Natl. Acad. Sci. 104: 7068-7073.

Mukherjee, S., Berger, M.F., Jona, G., Wang, X.S., Muzzey, D., Snyder, M., Young, R.A., and Bulyk, M.L. 2004. Rapid analysis of the DNA-binding specificities of transcription factors with DNA microarrays. Nat. Genet. 36: 1331-1339.

Newburger, D.E. and Bulyk, M.L. 2009. UniPROBE: An online database of protein binding microarray data on protein-DNA interactions. Nucleic Acids Res. 37: D77-D82.

Ng, H.H., Robert, F., Young, R.A., and Struhl, K. 2002. Genome-wide location and regulated recruitment of the RSC nucleosome-remodeling complex. Genes \& Dev. 16: 806-819.

Philippakis, A.A., Qureshi, A.M., Berger, M.F., and Bulyk, M.L. 2008. Design of compact, universal DNA microarrays for protein binding microarray experiments. J. Comput. Biol. 15: 655-665.

Pilpel, Y., Sudarsanam, P., and Church, G.M. 2001. Identifying regulatory networks by combinatorial analysis of promoter elements. Nat. Genet. 29: 153-159.

Pokholok, D.K., Harbison, C.T., Levine, S., Cole, M., Hannett, N.M., Lee, T.I., Bell, G.W., Walker, K., Rolfe, P.A., Herbolsheimer, E., et al. 2005. Genome-wide map of nucleosome acetylation and methylation in yeast. Cell 122: 517-527.

Pramila, T., Miles, S., GuhaThakurta, D., Jemiolo, D., and Breeden, L.L. 2002. Conserved homeodomain proteins interact with MADS box protein $\mathrm{Mcm} 1$ to restrict ECB-dependent transcription to the M/G1 phase of the cell cycle. Genes \& Dev. 16: 3034-3045.

Reece, R.J. and Ptashne, M. 1993. Determinants of binding-site specificity among yeast C6 zinc cluster proteins. Science 261: 909-911.

Ren, B., Robert, F., Wyrick, J.J., Aparicio, O., Jennings, E.G., Simon, I., Zeitlinger, J., Schreiber, J., Hannett, N., Kanin, E., et al. 2000. Genome-wide location and function of DNA binding proteins. Science 290: 2306-2309.

Robinson, M., Grigull, J., Mohammad, N., and Hughes, T. 2002. FunSpec: A web-based cluster interpreter for yeast. BMC Bioinformatics 3: 35. doi: 10.1186/1471-2105-3-35.

Roth, F.P., Hughes, J.D., Estep, P.W., and Church, G.M. 1998. Finding DNA regulatory motifs within unaligned noncoding sequences clustered by whole-genome mRNA quantitation. Nat. Biotechnol. 16: 939-945.

Simon, I., Barnett, J., Hannett, N., Harbison, C., Rinaldi, N., Volkert, T., Wyrick, J., Zeitlinger, J., Gifford, D., Jaakkola, T., et al. 2001. Serial regulation of transcriptional regulators in the yeast cell cycle. Cell 106: 697-708.

Tanay, A. 2006. Extensive low-affinity transcriptional interactions in the yeast genome. Genome Res. 16: 962-972.

Tanay, A., Regev, A., and Shamir, R. 2005. Conservation and evolvability in regulatory networks: The evolution of ribosomal regulation in yeast. Proc. Natl. Acad. Sci. 102: 7203-7208.

Tavazoie, S., Hughes, J., Campbell, M., Cho, R., and Church, G. 1999. Systematic determination of genetic network architecture. Nat. Genet. 22: 281-285.

Wei, C.L., Wu, Q., Vega, V.B., Chiu, K.P., Ng, P., Zhang, T., Shahab, A., Yong, H.C., Fu, Y., Weng, Z., et al. 2006. A global map of p53 transcriptionfactor binding sites in the human genome. Cell 124: 207-219.

Wilson, B., Erdjument-Bromage, H., Tempst, P., and Cairns, B.R. 2006. The RSC chromatin remodeling complex bears an essential fungal-specific protein module with broad functional roles. Genetics 172: 795-809.

Workman, C.T., Mak, H.C., McCuine, S., Tagne, J.B., Agarwal, M., Ozier, O., Begley, T.J., Samson, L.D., and Ideker, T. 2006. A systems approach to mapping DNA damage response pathways. Science 312: 1054-1059.

Received December 11, 2008; accepted in revised form January 14, 2009.

\section{Genome Research}

www.genome.org 


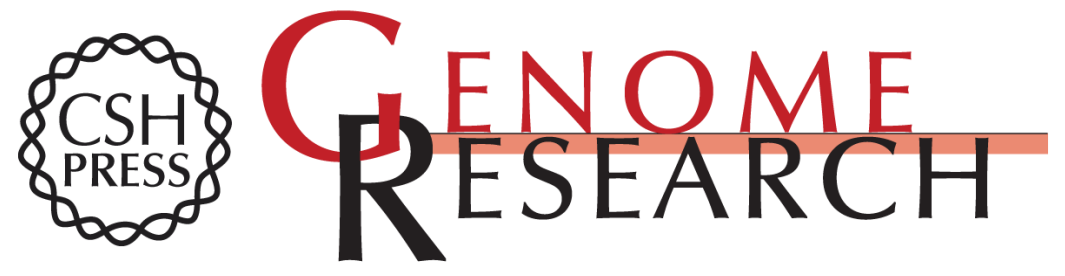

\section{High-resolution DNA-binding specificity analysis of yeast transcription factors}

Cong Zhu, Kelsey J.R.P. Byers, Rachel Patton McCord, et al.

Genome Res. 2009 19: 556-566 originally published online January 21, 2009

Access the most recent version at doi:10.1101/gr.090233.108

\section{Supplemental http://genome.cshlp.org/content/suppl/2009/03/05/gr.090233.108.DC1}

Material

References This article cites 56 articles, 23 of which can be accessed free at: http://genome.cshlp.org/content/19/4/556.full.html\#ref-list-1

Open Access Freely available online through the Genome Research Open Access option.

License Freely available online through the Genome Research Open Access option.

Email Alerting Receive free email alerts when new articles cite this article - sign up in the box at the Service top right corner of the article or click here.

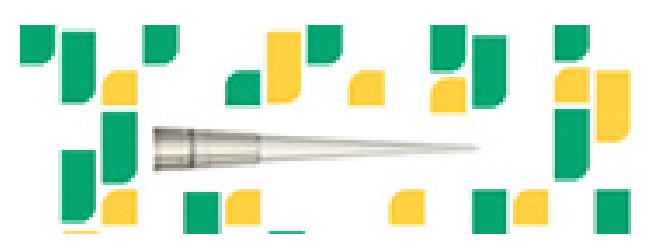

Focused on your science.

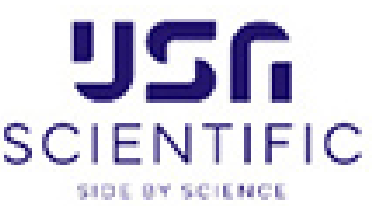

To subscribe to Genome Research go to:

https://genome.cshlp.org/subscriptions 